\title{
New Safety Information
}

National Cancer Institute

\section{Source}

National Cancer Institute. New Safety Information. NCI Thesaurus. Code C142613.

Pharmaceutical data received from factual, observational sources such as adverse event reports, clinical or post-approval studies or other scientific studies about a severe or unexpected severe hazard related to the pharmaceutical after approval or following the most recent assessment of the drug. 